\title{
Repair of Osteoporotic Bone Defects Using Adipose-Derived Stromal Cells and Umbilical Vein Endothelial Cells Seeded in Chitosan/Nanohydroxyapatite-P24 Nanocomposite Scaffolds
}

\author{
Yifei Fang $\mathbb{D}^{1},{ }^{1}$ Yong Gong $\mathbb{D},{ }^{2}$ Zhijian Yang $\mathbb{D}^{1},{ }^{1}$ and Yan Chen $\mathbb{D}^{3}$ \\ ${ }^{1}$ Department of Orthopedics, Zhujiang Hospital, Southern Medical University, Guangzhou 510282, China \\ ${ }^{2}$ Department of Orthopedics, The Sixth Affiliated Hospital, Sun Yat-sen University, Guangzhou 510655, China \\ ${ }^{3}$ Department of Ultrasonic Diagnosis, Zhujiang Hospital, Southern Medical University, Guangzhou 510282, China \\ Correspondence should be addressed to Yan Chen; smu_chen@163.com
}

Received 27 May 2021; Accepted 29 July 2021; Published 21 August 2021

Academic Editor: Hui Qi Xie

Copyright (c) 2021 Yifei Fang et al. This is an open access article distributed under the Creative Commons Attribution License, which permits unrestricted use, distribution, and reproduction in any medium, provided the original work is properly cited.

\begin{abstract}
Background. The cell regeneration and blood supply of bone defect lesions are restricted under osteoporotic pathological conditions, which make the healing of bone defect of osteoporosis still a great challenge. The current therapeutic strategies that mainly inhibit bone resorption are not always satisfactory for osteoporotic bone defects, which make the development of new therapies an urgent need. Methods. Previously, we prepared chitosan/nanohydroxyapatite (CS/nHA) biomimetic nanocomposite scaffolds for controlled delivery of bone morphogenetic protein 2-derived peptide (P24). In this study, we determined the effect of coculturing adipose-derived stromal cells (ADSCs) and human umbilical vein endothelial cells (HUVECs) with the CS-P24/nHA nanocomposite scaffolds on osteoporotic bone defect healing. In vitro mixed coculture models were employed to assess the direct effects of coculture. Results. ADSCs cocultured with HUVECs showed significantly greater osteogenic differentiation and mineralization compared with ADSCs or HUVECs alone. The CS-P24/nHA scaffold cocultured with ADSCs and HUVECs was more effective in inducing osteoporotic bone repair, as demonstrated by micro-computed tomography and histology of critical-sized calvariae defects in ovariectomized rats. Calvariae defects treated with the CS-P24/nHA nanocomposite scaffold plus ADSC/HUVEC coculture had a greater area of repair and better reconstitution of osseous structures compared with defects treated with the scaffold plus ADSCs or the scaffold plus HUVECs after 4 and 8 weeks. Conclusion. Taken together, coculture of ADSCs and HUVECs with the CS-P24/nHA nanocomposite scaffold is an effective combination to repair osteoporotic bone defects.
\end{abstract}

\section{Introduction}

Osteoporosis is a metabolic systemic bone disease characterized by decreased bone mass, increased bone fragility, and weakened bone strength [1]. More than 8 million fractures are caused by osteoporosis every year worldwide, and osteoporosis is the leading cause of fracture in older women [2]. More than one-third of older women experience osteoporosis-induced fracture [2]. Antibone resorption drugs are the most widely used in the clinic, including parathyroid hormone, bisphosphonate, raloxifene, and dinozumab [3, 4]. These drugs exert therapeutic effects by inhibiting osteoclast activity and bone resorption; however, they do not influence bone regeneration, so it is difficult to reconstruct bone using these approaches $[5,6]$. For patients with osteoporotic bone defects, due to the significantly weakened ability of mesenchymal stem cell (MSC) regeneration and osteogenic differentiation, antibone resorption therapy alone is insufficient; thus, bone regeneration therapy is required [7, 8]. Existing bone defect implants, such as autogenous bone and artificial bone, are considered to lack sufficient biological activity to induce differentiation of endogenous MSCs into osteoblasts in patients with osteoporosis. They also have an insufficient effect on osteoporotic bone regeneration [9]. Therefore, there is an urgent need to improve bone defect implants to achieve osteoporotic bone regeneration. 
Although biological implants have more advantages compared with autogenous bone, they do have shortcomings such as ischemic necrosis and apoptosis of implanted cells in the early stage of transplantation, which are caused by an insufficient blood supply [10]. In bone tissue engineering, neovascularization can facilitate oxygen and nutrient exchange between implants and cells, which is important for the survival and differentiation of stem cells in scaffolds [11, 12]. Studies have shown that microvessel formation is a prerequisite for bone formation in implants [11]. Whether it is the exchange of oxygen and nutrients or the calcium and phosphorus needed for mineralization, a complete vascular network is essential [13].

Methods to promote microvessel growth include the use of cytokines, such as vascular endothelial growth factor (VEGF) and basic fibroblast growth factor (bFGF), as well as endothelial cell transplantation [11]. Cytokines are expensive, and their release and onset of effects in the body are difficult to control, so endothelial cells are preferred [14]. Endothelial cells are the cellular source of microangiogenesis and play an important role in vasculogenesis and angiogenesis [15]. Moreover, endothelial cells can be simply extracted from peripheral blood, which not only causes little damage to the donor but also reduces the likelihood of rejection after autologous cell transplantation [15]. Implantation of endothelial cells into the scaffold can provide nutritional support to the vasculature for osteogenic differentiation of stem cells. Previous studies have shown that coculturing endothelial cells and stem cells in the scaffold can induce graft blood vessel formation and achieve vascularization and osteogenesis simultaneously $[12,13,15,16]$. Therefore, efforts to establish a system to coculture endothelial cells and stem cells in bone tissue engineering have been made. To apply the concept of endothelial cell and stem cell coculture in the clinic, it is important to determine whether endothelial cells and stem cells are beneficial or harmful in osteogenic differentiation and angiogenesis when used to treat bone defects.

Sulfhydrylated chitosan (CS) is obtained by amino modification of CS [17]. Compared with ordinary CS, sulfhydrylated CS has better adhesion, stability, and protein release properties [18]. The CS/nanohydroxyapatite (HA) nanocomposite scaffold prepared using sulfhydrylated CS and nanohydroxyapatite has widespread application potential in cell colonization and factor release because of its porous structure, excellent biocompatibility, and moderate degradation rate [19]. P24 is a peptide derived from bone morphogenetic protein (BMP-2). It has a low molecular weight and a stable structure, as well as similar biological effects to BMP2 [19]. P24 can regulate the adhesion and osteogenic differentiation of MSCs, promote deposition of calcium and phosphate ions, and accelerate nucleation and mineralization [20]. Due to the high therapeutic dose of BMP-2 in clinical application, it is easy to lead to complications such as excessive bone resorption, heterotopic ossification, and tumor angiogenesis [21]. P24, which has similar biological effects and is more absorbable, is an excellent alternative to BMP2. In a previous study, CS/nHA nanocomposite scaffold was prepared using chemical grafting modification technology, and this scaffold was used to control P24 delivery [19]. The results show that the scaffold has a good effect on repairing bone defects in rats. To further expand the application of this CS/nHA nanocomposite scaffold in the treatment of osteoporotic bone defects, we intend to use cell coculture to further enhance the ability of this scaffold to repair bone defects.

The purpose of this study was to investigate the effect of adipose-derived stromal cell (ADSC) and human umbilical endothelial cell (HUVEC) coculture with the CS-P24/nHA nanocomposite scaffold in repairing osteoporotic bone defects. We also aimed to explore the application prospects of this coculture system.

\section{Methods}

2.1. Materials. Chitosan (CS, viscosity: $50-800 \mathrm{mPa} \cdot \mathrm{s}$, degree of deacetylation: $80 \%-95 \%)$ was purchased from Macklin, China. Peptide 24 (P24) derived from BMP-2 $(\mathrm{N} \rightarrow \mathrm{C}$ : KIPKA SSVPT ELSAI STLYL SGGC) was synthesized by Shanghai ZiYu Biotech Co., China. 2-Iminothiolane hydrochloride, 2-iminothiolane hydrochloride, and dimethyl sulfoxide (DMSO) were purchased from Sigma-Aldrich, USA. nHA powder was obtained from Institute of Nuclear and New Energy Technology, Tsinghua University, China.

2.2. Culture of ADSCs and HUVECs. Human ADSCs were isolated following established methods. The procedure was approved by the Research Committee of South Medical University. Briefly, adipose tissue samples were collected from patients undergoing reconstructive surgery. Adipose tissue was digested in 50-200 U/ml collagenase type I (SigmaAldrich, USA) for $2 \mathrm{~h}$. The suspension was oscillated at a constant temperature of $37^{\circ} \mathrm{C}$ for 2 hours and then centrifuged at $5000 \mathrm{~g}$ for 10 minutes. The cells obtained after centrifugation were cultured in expansion medium consisting of Dulbecco's modified Eagle's medium/F-12, 10\% fetal bovine serum (FBS; Gibco BRL), and 1\% penicillin/streptomycin. HUVECs were obtained from the American Type Culture Collection (Rockville, MD, USA) and cultured in RPMI 1640 medium (Gibco, USA) containing 10\% FBS and $1 \%$ penicillin/streptomycin [1]. Cells at passages 3-4 were used in this study. Cells were cultured under standard conditions $\left(5 \% \mathrm{CO}_{2}, 37^{\circ} \mathrm{C}\right)$.

2.3. Scaffold Synthesis and ADSC/HUVEC Coculture. Scaffolds containing CS with 0\% P24 and nHA (CS-0\%P24/ nHA) and scaffolds containing CS with 10\% P24 and nHA (CS-10\%P24/nHA) were prepared as outlined previously [19]. Briefly, $40 \mathrm{mg}$ of 2-iminothiolane hydrochloride was added to $200 \mathrm{ml}$ of $0.2 \%(w / v)$ chitosan solution (in $1 \%$ acetic acid). P24 ( $0 \%$ or $10 \%$ of the weight of chitosan in $15 \mathrm{ml}$ DMSO) was then added into the chitosan solution. After continuous stirring at room temperature for $4 \mathrm{~h}$, the mixture was dialyzed against deionized water for 5 days and lyophilized at $-50^{\circ} \mathrm{C}$ and $20 \mathrm{~Pa}$ in order to immobilize the peptide within the chitosan (chitosan-peptide 24, CS-0\%P24, and CS-10\%P24).

$200 \mathrm{mg}$ of CS-0\%P24 and CS-10\%P24 were hydrated in $10 \mathrm{ml} 0.1 \mathrm{M} \mathrm{HCl}$, respectively. nHA powder $(200 \mathrm{mg})$ was added with continuous stirring until uniformly distributed. 
Thereafter, the hybrid of CS-P24 and HA was lyophilized at $-50^{\circ} \mathrm{C}$ and $20 \mathrm{~Pa}$ using a 96-well plate (Corning, USA) as a mold to obtain the CS-0\%P24/HA and CS-10\%P24/HA scaffold.

With the CS-10\%P24/nHA scaffold cultured with ADSCs and HUVECs, these two cell types were trypsinized separately and subcultured in 24-well plates at ratios of $1: 1$, $1: 3$, and $3: 1$ during the same seeding phase. The CS$10 \% \mathrm{P} 24 / \mathrm{nHA}$ scaffold cultured with ADSCs or HUVECs served as a control.

2.4. Cell Proliferation Assay. Cell proliferation was measured using the Cell Counting Kit-8 (CCK-8; Dojindo). With the CS-10\%P24/nHA scaffold cultured with ADSCs and HUVECs, cells were trypsinized separately and subcultured in 96-well plates at ratios of $1: 1,1: 3$, and $3: 1$. Each group has 6 experimental samples. After 1,3, and 7 days, cells in each group were incubated in $10 \%$ CCK- 8 solution in a $5 \%$ $\mathrm{CO}_{2}$ incubator at $37^{\circ} \mathrm{C}$ for $4 \mathrm{~h}$. The absorbance of the culture medium was measured at $450 \mathrm{~nm}$.

2.5. Alizarin Red S Staining. Alizarin Red S staining and quantification were performed as described previously [22]. Cells in different groups were stained 21 days after culture, and each group has 6 experimental samples. Cells were fixed in $4 \%$ paraformaldehyde (Leagene, China) for $15 \mathrm{~min}$. After being fixed, cells were stained with $1 \%$ Alizarin Red S for $30 \mathrm{~min}$. Stained samples were air dried, and images were taken using an optical microscope (Leica, DM2500 LED).

2.6. Real-Time Quantitative PCR. After 10 days of coculture, total RNA was isolated by lysis in TRIzol (Invitrogen Inc., Carlsbad, CA, USA). PCR was performed using the Transcriptor cDNA Synthesis Kit and FastStart Universal SYBR Green Master (Roche Diagnostics, Indianapolis, IN, USA). The mRNA expressions of osteogenic-specific genes (OCN, ALP, and RUNX2) were assessed by real-time quantitative PCR using SYBR Green Master (Roche Diagnostics). The sequences of all primers (Invitrogen Inc.) were designed using Primer 5.0 software (Table 1). $\beta$-Actin was amplified as an internal control. Each real-time quantitative PCR run was performed using three experimental samples. Error bars reflect one standard deviation from the mean of technical replicates.

\subsection{In Vivo Calvaria Defect Repair}

2.7.1. Osteoporotic Rat Model and Transplantation Procedure. To generate osteoporotic rat models, 36 female SpragueDawley rats $(200-220 \mathrm{~g})$ at 7 weeks of age $(n=6)$ were purchased from the Center of Experimental Animals of Guangdong Province. Rats were ovariectomized bilaterally with a dorsal approach. After 4 weeks, under general anesthesia, the cranium was exposed through a medial incision. Bilateral full-thickness circular defects of $5 \times 5 \mathrm{~mm}$ were generated using a dental bur. The defects were implanted with CS-0\%P24/nHA, CS-10\%P24/nHA, CS-10\%P24/nHA with ADSCs, CS-10\%P24/nHA with HUVECs, or CS$10 \% \mathrm{P} 24 / \mathrm{nHA}$ with ADSC/HUVEC coculture. The coculture ration of ADSCs and HUVECs was $3: 1$. The control groups
TABLE 1: Forward and reverse primer sequences used in real-time PCR analysis.

\begin{tabular}{lcc}
\hline Gene & & Primer $\left(5^{\prime}-3^{\prime}\right)$ \\
\hline \multirow{2}{*}{ OCN } & Forward primer & GCTCTGTGCTCCTGCATCTG \\
& Reverse primer & GCTCTGTGCTCCTGCATCTG \\
\multirow{2}{*}{ ALP } & Forward primer & ACCATTCCCACGTCTTCACATTT \\
& Reverse primer & AGACATTCTCTCGTTCACCGCC \\
\multirow{2}{*}{ RUNX2 } & Forward primer & GCCTTCAAGGTGGTAGCCC \\
& Reverse primer & CGTTACCCGCCATGACAGTA \\
\hline
\end{tabular}

were left untreated. In all animals, the wound was irrigated, and the fascia and skin were closed. Postoperatively, animals were allowed free cage activity. To establish an osteoporosis model, the rats were injected with cyclosporine A ( $5 \mathrm{mg} / \mathrm{kg}$ of body weight) and methylprednisolone hemisuccinate ( $1 \mathrm{mg} / \mathrm{kg}$ body weight) daily after surgery. Whole calvariae were harvested for evaluation 4 and 8 weeks of postimplantation.

2.7.2. Micro-Computed Tomography (CT) Analysis of Calvaria Defect Repair. After harvesting calvariae at 4 and 8 weeks postoperatively, specimens were immediately fixed in $10 \%(v / v)$ neutral-buffered formalin for $24 \mathrm{~h}$. Specimens were scanned at a resolution of $9 \mu \mathrm{m}$ for undecalcified samples using an advanced micro-CT instrument (ZKKSMC-Sharp-IV; Zhongke Kaisheng Bio, Inc.). Threedimensional reconstruction of images was performed, and histomorphometric parameters, including bone mineral density (BMD), trabecular number (Tb.N), and bone volume/total tissue volume (BV/TV), were evaluated.

2.7.3. Immunofluorescence, Immunohistochemistry, and Histomorphometry. Specimens were decalcified in neutral $10 \%$ EDTA solution for 2 weeks at room temperature. Slices were then cut into $6 \mu \mathrm{m}$ sections and further stained with anti-OCN (1:500 dilution; Abcam) and anti-CD31 (1:600 dilution; Abcam), respectively, at $4^{\circ} \mathrm{C}$ overnight, followed by horseradish peroxidase-conjugated goat anti-rabbit secondary antibodies (Boster Company of Biotechnology, China). Images of stained specimens were obtained using a microscope (BX51; Olympus, Tokyo, Japan). The expression intensity of each molecule was quantified using Image J analysis software (1.8.0 64-bit, US National Institutes of Health).

For immunofluorescence analysis, slides or chondrocytes were fixed in $4 \%$ buffered formalin and incubated with fluorescence-conjugated secondary antibody for $90 \mathrm{~min}$ at room temperature. Sections were also stained with hematoxylin and eosin, Masson's trichrome, or tartrate-resistant acid phosphatase (TRAP).

2.7.4. Cell Tracking Using Anti-Human Nuclear Antibody. Sections from each group at 4 and 8 weeks were used postoperatively for cell tracking analysis. Sections were stained with mouse anti-human nuclei monoclonal antibody (MAB1281; 1:500 dilution; Merck Millipore, Germany) for immunofluorescence and immunohistochemistry. MAB1281-labeled cells were marked. 


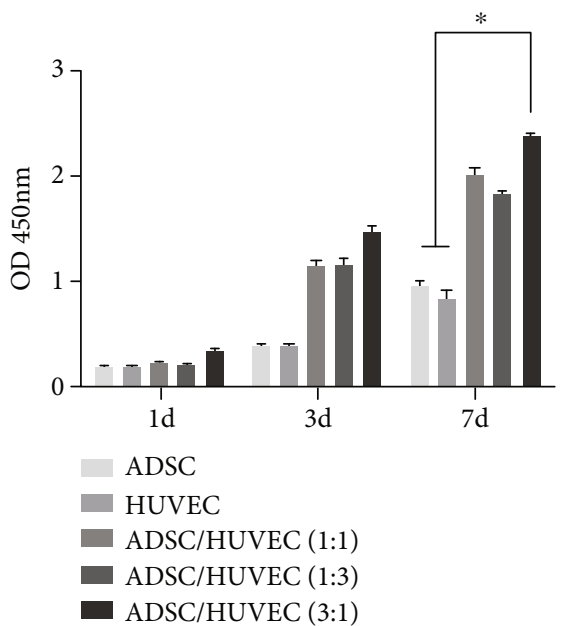

(a)
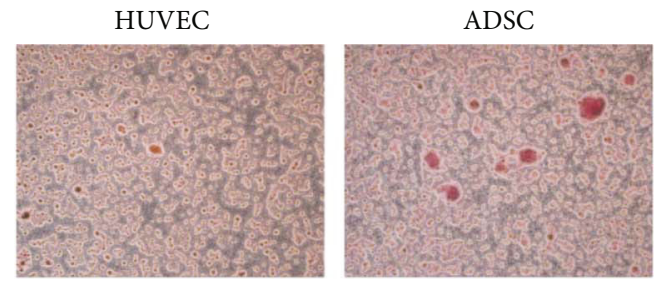

$1: 1$

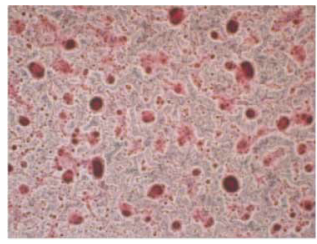

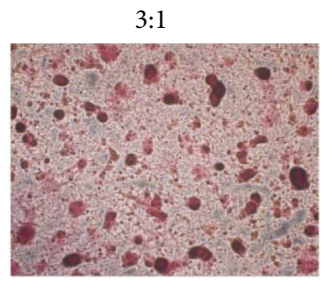

ALP

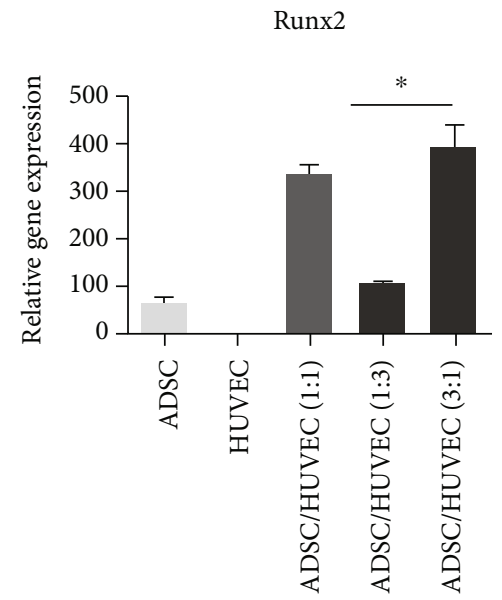

OCN


(c)

FIGURE 1: In vitro osteogenesis and angiogenesis. ASC/HUVEC ratios of $1: 1,1: 3$, and $3: 1$ were achieved by culturing $1 \times 10^{5}, 0.3 \times 10^{5}$, and $3.0 \times 10^{5}$ ADSCs, respectively, with $10^{5}$ HUVECs. (a) The CCK- 8 test of cell viability after coculture for 1,3 , and 7 days. (b) Alizarin Red S staining after 14 days. All values are shown as percentages compared with control. (c) mRNA expression of osteogenic-specific genes (ALP, $O C N$, and RUNX2) in each group at 10 days. mRNA expression, quantified using real-time quantitative PCR, was normalized to $\beta$-actin as a reference gene. ${ }^{*} p<0.05$. Error bars represent the standard deviation $(n=3)$.

2.8. Statistical Analysis. Descriptive statistics were used to determine group means and standard deviations. Quantitative data were statistically analyzed using Student's $t$-test (SPSS 22.0). A $p$ value of $<0.05$ was considered statistically significant.

\section{Results}

3.1. In Vitro Osteogenesis. To determine the effect of coculture on cell growth, ADSCs, HUVECs, or ADSCs plus
HUVECs at ratios of $1: 1,1: 3$, and $3: 1$ were seeded onto CS-10\%P24/nHA scaffolds. Cell proliferation was measured using the CCK- 8 assay. The CCK- 8 analysis showed that cell number significantly increased in each of the ADSC, HUVEC, and ADSC/HUVEC coculture groups. No matter what the ratio of cells was, the proliferation rate in the ADSC/HUVEC coculture group was significantly higher than that in the ADSC and HUVEC groups. When the ratio of ADSCs to HUVECs was $3: 1$, the proliferation rate was the most significant, and the absorbance on day 7 was about 2.4 


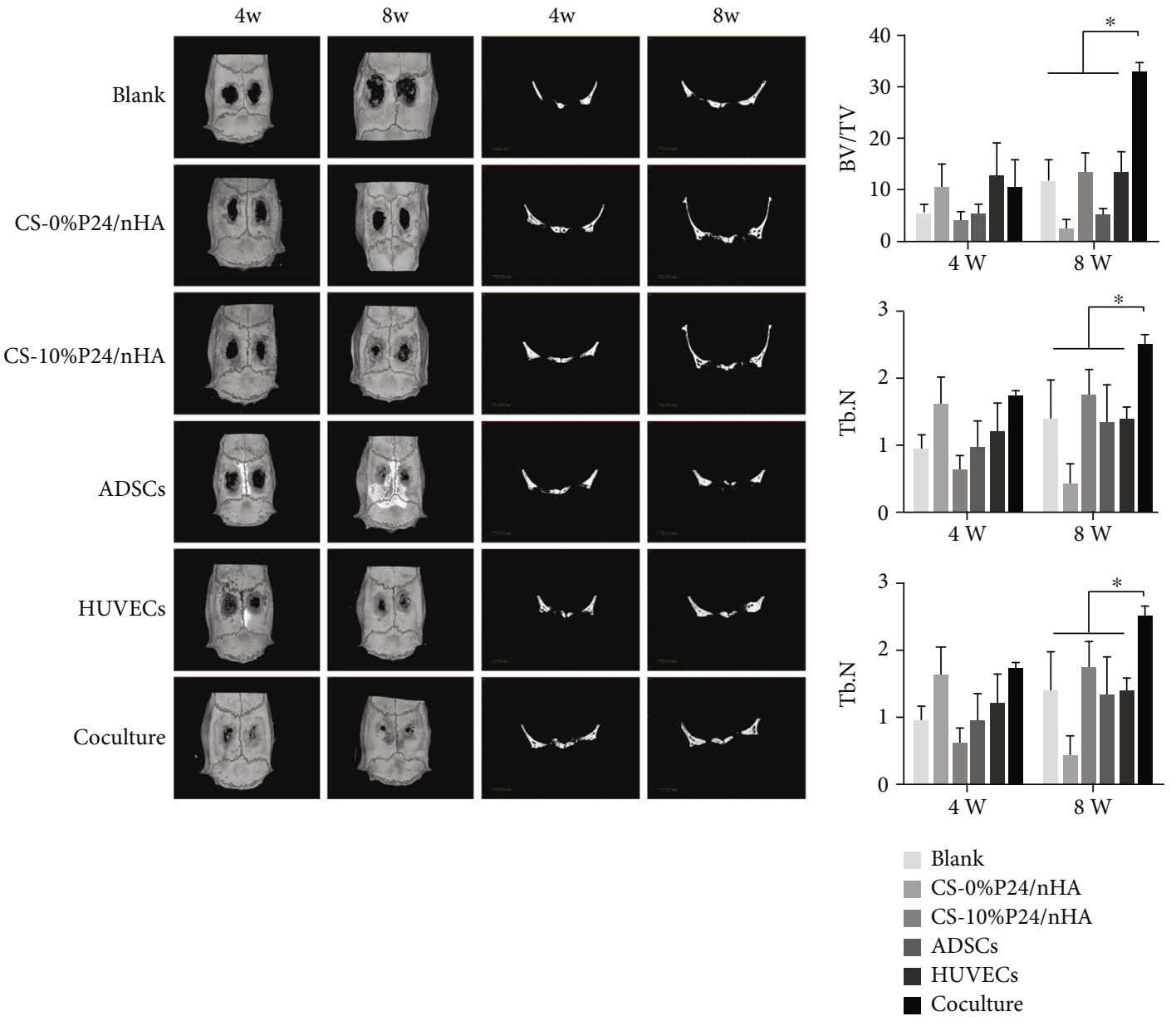

(a)

(b)

FIGURE 2: In vivo effects of the scaffold plus ADSC/HUVEC coculture on osteoporotic bone formation in critical-sized calvariae defects. Two circular defects, each with a diameter of $3 \mathrm{~mm}$, were created using a trephine bur in mice calvariae. Defects were filled with a CS-0\%P24/nHA scaffold, a CS-10\%P24/nHA scaffold, or a CS-10\%P24/nHA scaffold with HUVECs and/or ADSCs. (a) Reconstructed three-dimensional micro-CT images at 4 and 8 weeks in each group. Micro-CT of whole calvariae in each group, indicating new bone formation at the defect site. (b) Quantitative analysis of mineralized new bone formation at 4 and 8 weeks (BMD, Tb.N, and BV/TV). ${ }^{*} p<0.05$.

and 2.7 times of that in the ADSC and HUVEC groups (Figure 1(a)).

Alizarin Red S staining was used to evaluate calcium deposition in each group. HUVECs cultured alone appeared light red in color, ADSCs cultured alone appeared as a deeper red color, while cocultured ADSCs/HUVECs appeared as a very deep red color. Although mineralized nodules were observed in all three groups, more distinct nodules were observed in the ADSC/HUVEC coculture group on day 21 (Figure 1(b)).

To evaluate the effect of the CS-P24/nHA scaffold cultured with ADSCs, HUVECs, or ADSCs plus HUVECs on osteogenic differentiation, we performed real-time quantitative PCR to examine osteogenic gene expression. RUNX2, OCN, and $A L P$ were significantly upregulated in the ADSC/HUVEC coculture group (Figure 1(c)). When the ratio of ADSCs to HUVECs was $3: 1$, the expression levels of RUNX2, OCN, and ALP were the highest among all groups.

These results suggested that when ADSCs and HUVECs were cocultured at a ratio of $3: 1$, the degree of cell prolifera- tion and osteogenic differentiation was the most significant. Therefore, in subsequent experiments, 3:1 was used as the cell ratio of ADSC and HUVEC coculture.

3.2. In Vivo Effects of ADSC/HUVEC Coculture on Osteoporotic Bone Formation. In vivo osteoporotic bone formation was evaluated by micro-CT at 4 and 8 weeks of postimplantation (Figure 2). When CS-10\%P24/nHA scaffolds were used to treat critical-sized calvariae defects (control group), most osteoporotic bone defects were not repaired after 8 weeks (Figure 2(a)). Scaffolds with HUVECs alone, ADSC/HUVEC coculture, and ADSCs alone significantly increased the repaired area $(p<0.05)$ compared with the control scaffold (Figure 2(b)). Defects treated with the scaffold plus ADSC/HUVEC coculture had significantly greater BMD $(442.50 \pm 15.00)$, Tb.N $(2.52 \pm 0.25)$, and $\mathrm{BV} / \mathrm{TV}(33.00 \pm 3.28)$ values compared with the other groups $(p<0.05)$.

Histological findings showed that calvariae defects treated with the scaffold plus ADSC/HUVEC coculture had 




(a)

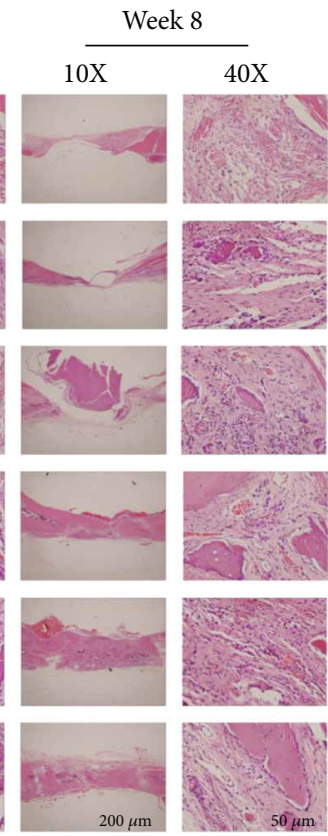

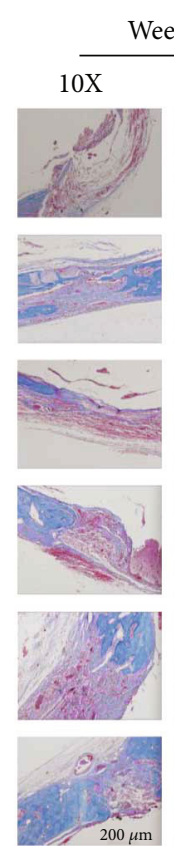

eek 4 $40 \mathrm{X}$
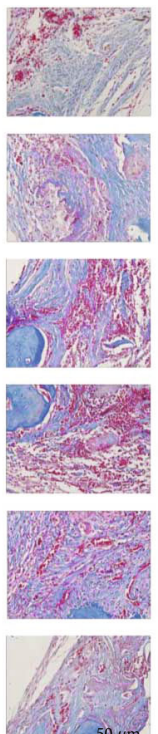
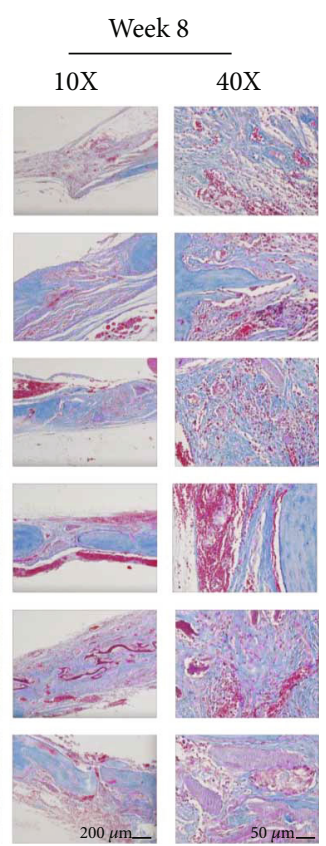

(b)

FIGURE 3: Histology of repaired calvariae 4 and 8 weeks of postimplantation in vivo. (a) Hematoxylin and eosin staining of calvariae defects in the blank, CS-0\%P24/nHA, CS-10\%P24/nHA, CS-10\%P24/nHA plus ADSC, CS-10\%P24/nHA plus HUVEC, and CS-10\%P24/nHA plus ADSC/HUVEC coculture groups. Red arrows show new blood vessel formation in or around the scaffolds. (b) Masson's trichrome staining of repaired calvariae in each group. Scale bar $=200 \mu \mathrm{m}$ and $50 \mu \mathrm{m}$.

a thicker regenerated calvarium and better osseous structure reconstitution compared with those treated with the scaffold, HUVECs, or ADSCs alone (Figure 3). HE staining showed that osteoid cells and microvascular regeneration began to appear in the coculture group 4 weeks after surgery. At 8 weeks postoperatively, the mineralized tissue was filled into the scaffold and new microvessels could be observed (Figure 3(a)). Masson staining showed that fibrotic tissues developed earlier in the coculture group at week 4 and were gradually replaced by new bone tissue at week 8 (Figure 3(b)). These results indicated faster bone regeneration and better osseous structure reconstitution in the coculture group compared to the other groups.

Immunofluorescence and immunohistochemical analyses showed that the area implanted with the scaffold plus ADSC/HUVEC coculture exhibited high expression of OCN and CD31 at 4 and 8 weeks of postimplantation (Figure 4). The mean fluorescence intensity of CD31 and OCN in the coculture group was 2.5 and 2.2 times higher than that in the blank group, respectively (Figure 4(a)). In addition, the number of CD31-positive cells in the coculture group at 4 and 8 weeks was also significantly higher than that in the other groups, which was 4 and 3.5 times of that in the blank group, respectively (Figure 4(b)).

\subsection{Immunohistological Assessment of ADSC/HUVEC} Survival In Vivo. The survival of grafted cells was evaluated by immunohistochemistry using MAB1281. At 4 weeks of postimplantation, immunofluorescence and immunohistochemistry analyses revealed that MAB1281-labeled cells sur- vived within the scaffolds in the group that received the scaffold plus ADSCs and the group that received the scaffold plus ADSC/HUVEC coculture (Figure 5(a)). However, at 8 weeks of postimplantation, only the group with the scaffold plus ADSC/HUVEC coculture showed MAB1281-labeled cells (Figure 5(b)). These results indicate that the scaffold plus ADSC/HUVEC coculture can improve the survival rate of grafted cells.

3.4. Effect of the Scaffold plus ADSC/HUVEC Coculture on Osteoclastogenesis. We investigated the effect of the scaffold plus ADSC/HUVEC coculture on osteoclast formation in an osteoporotic environment. TRAP staining revealed that multiple osteoclasts lined the eroded bone surface in the blank group and in the group that received the CS$10 \% \mathrm{P} 24 / \mathrm{nHA}$ scaffold (Figure 6(a)). The erosion surface was reduced in the group that received the scaffold plus ADSCs and the group that received the scaffold plus HUVECs, and the number of osteoclasts was significantly lower in the group treated with the scaffold plus ADSC/HUVEC coculture $(4.60 \pm 0.57$ cell) (Figure 6(b)). Osteoclast formation was also inhibited in the group that received the scaffold plus ADSC/HUVEC coculture.

\section{Discussion}

It is generally believed that there is a close relationship between angiogenesis and osteogenesis [11, 23-26]. In the process of articular cartilage degeneration, hypertrophic chondrocytes enable microvessels to grow into the growth 


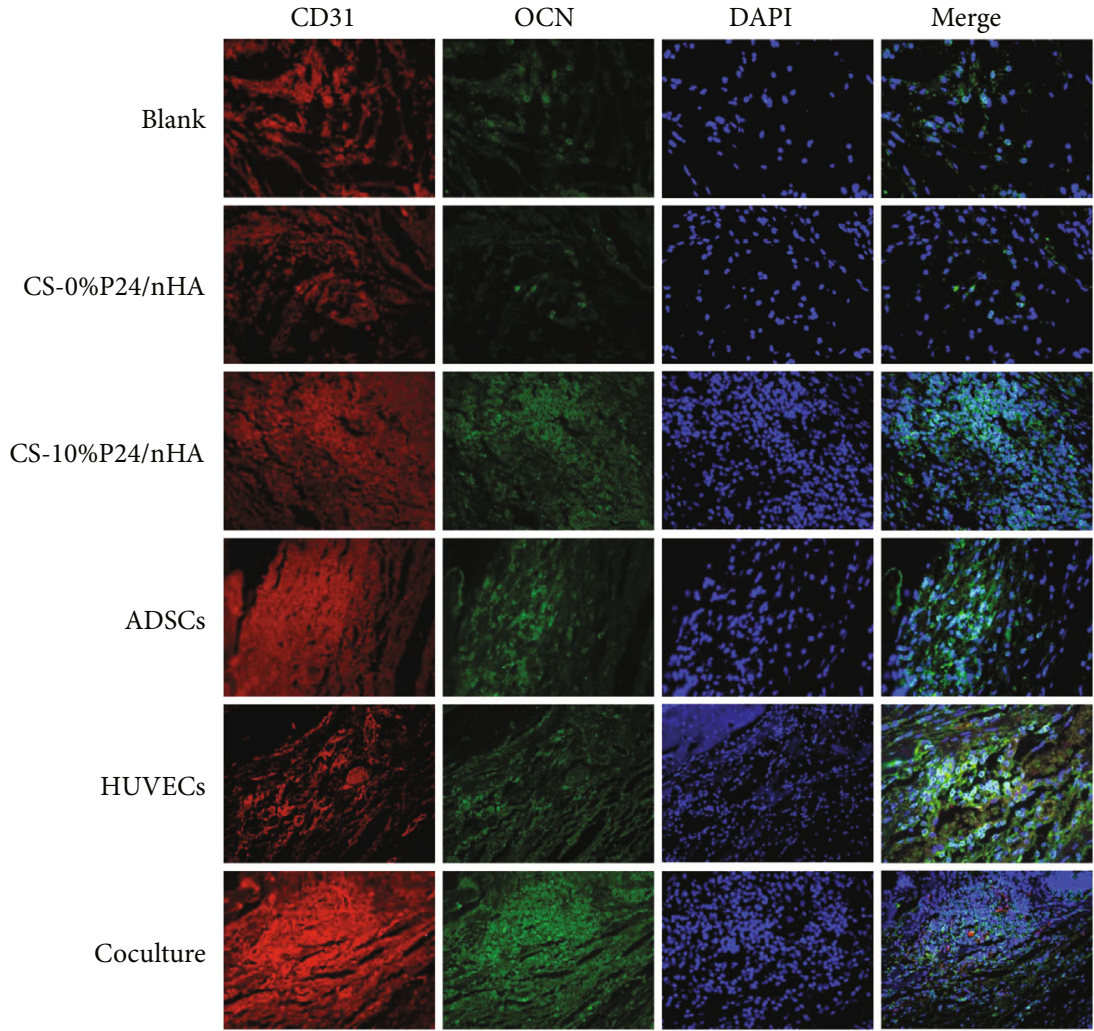

(a)
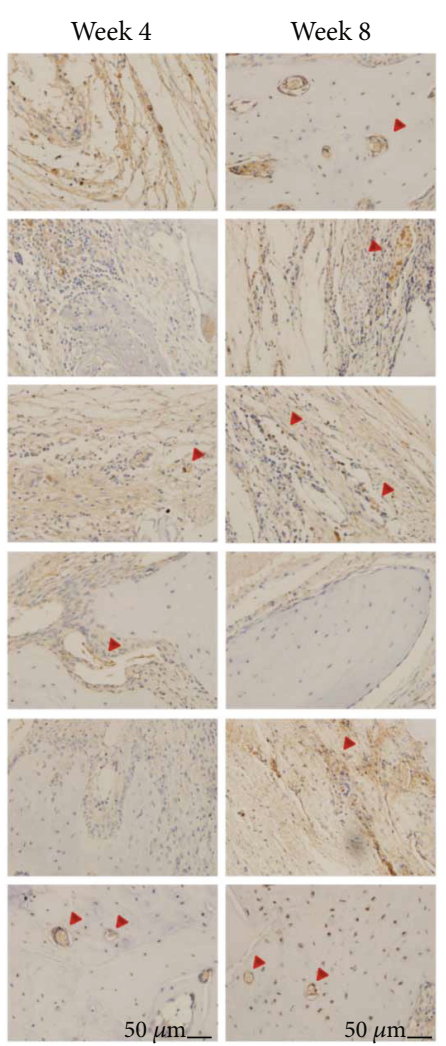

(b)

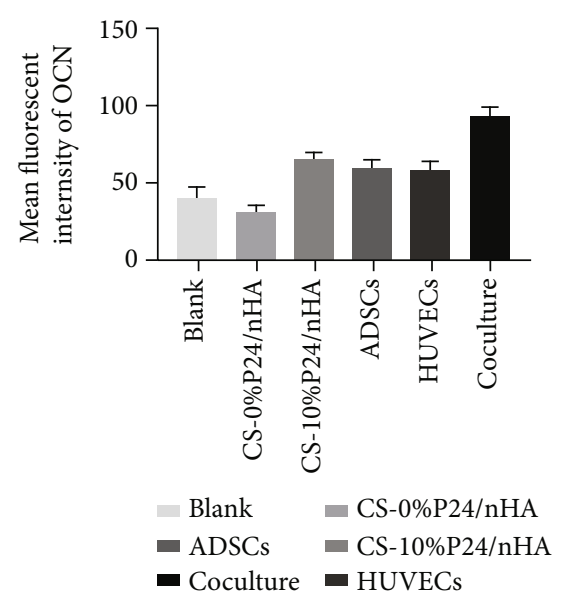

(c)

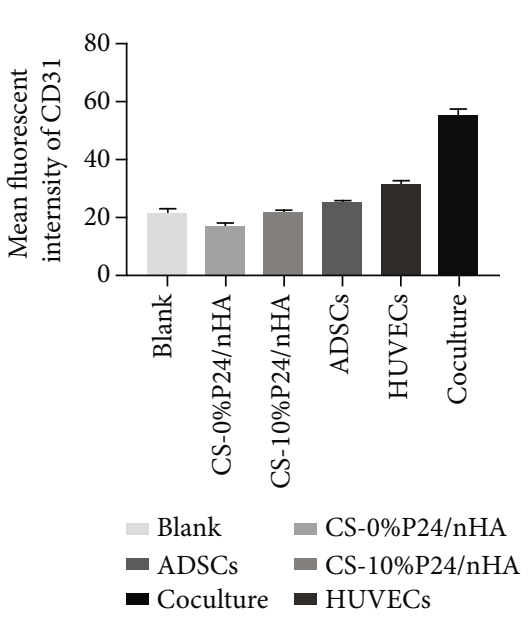

(d)

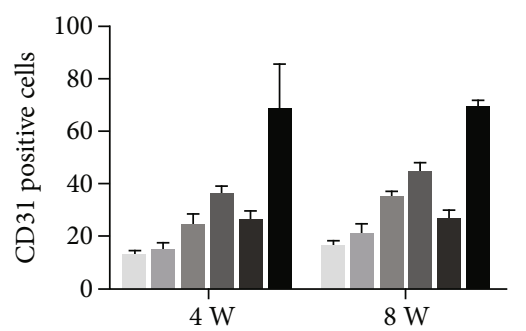

$\begin{array}{ll}\text { Blank } & \text { CS-0\%P24/nHA } \\ \text { - ADSCs } & \text { CS-10\%P24/nHA } \\ \text { - Coculture } & \text { HUVECs }\end{array}$

(e)

FIGURE 4: Immunofluorescence and immunohistochemistry. (a) Immunofluorescence of each group 4 weeks after surgery. Cells that appear green represent OCN-positive cells. Areas that appear red represent CD31-positive areas. (b) Immunohistochemistry of 4 and 8 weeks after surgery. Areas that appear dark brown represent CD31-positive areas, which indicate blood vessels (red arrow). The white areas are voids. (c) Mean fluorescent intensity of OCN-positive cells at 4 weeks. (d) Mean fluorescent intensity of CD31-positive cells at 4 weeks. (e) Quantification of CD31-positive cells in each group. Representative magnified images are at $\times 40$ magnification. Scale bar $=50 \mu \mathrm{m}$.

plate by secreting angiogenic factors, such as VEGF, which leads to cartilage absorption and bone formation [27, 28]. During bone repair, microvessels provide a prerequisite for the exchange of oxygen and nutrients for bone regeneration [23]. Lack of blood vessels will delay bone regeneration and can even lead to implant ischemic necrosis, delayed healing, and nonunion $[11,23]$. In patients with osteoporosis, it is more difficult to repair the bone defect because of active bone resorption and inhibition of osteogenesis $[8,29]$. Therefore, providing adequate vascular support for bone repair is helpful to facilitate bone regeneration, which is of great importance for the treatment of patients with osteoporotic bone defects. As the initiation and maintenance cells of vascular regeneration, endothelial cells play an indispensable role in 


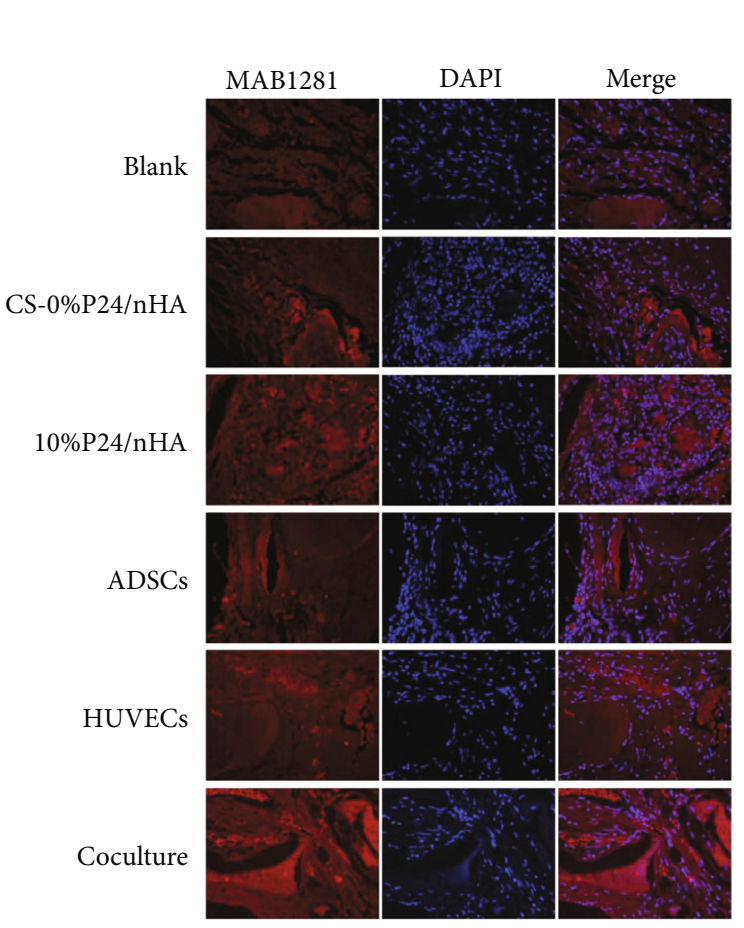

(a)

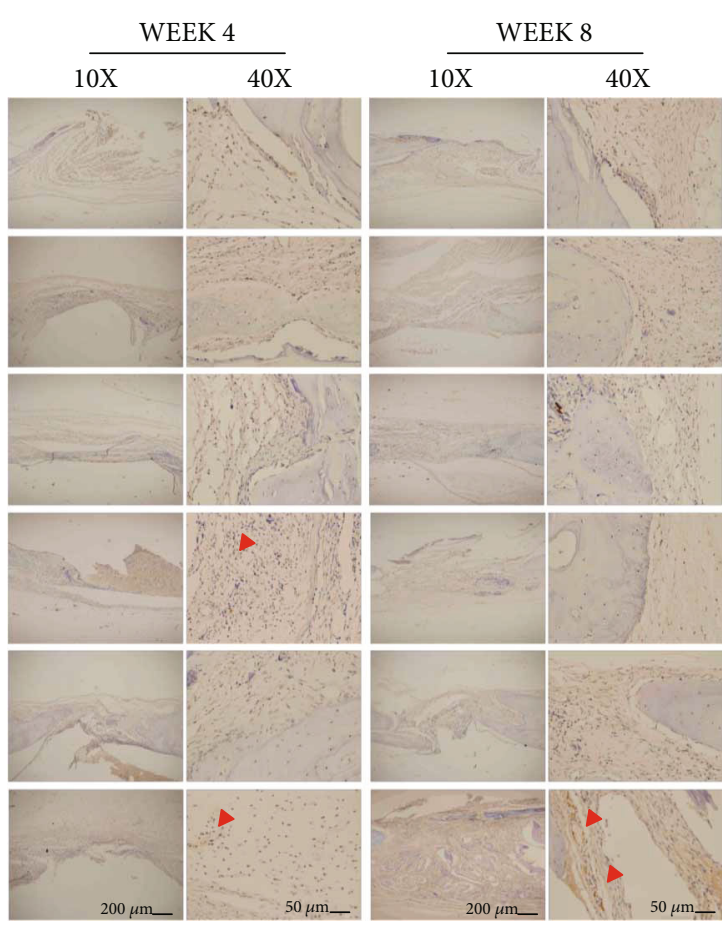

(b)

FIGURE 5: Survival of ADSCs/HUVECs in the scaffold. (a) Immunofluorescence and immunohistochemistry show MAB1281-labeled cells in the scaffold at 4 weeks. Areas that appear dark brown represent MAB1281-labeled areas. (b) Red arrows show MAB1281-labeled cells. Scale bar $=200 \mu \mathrm{m}$ and $50 \mu \mathrm{m}$.

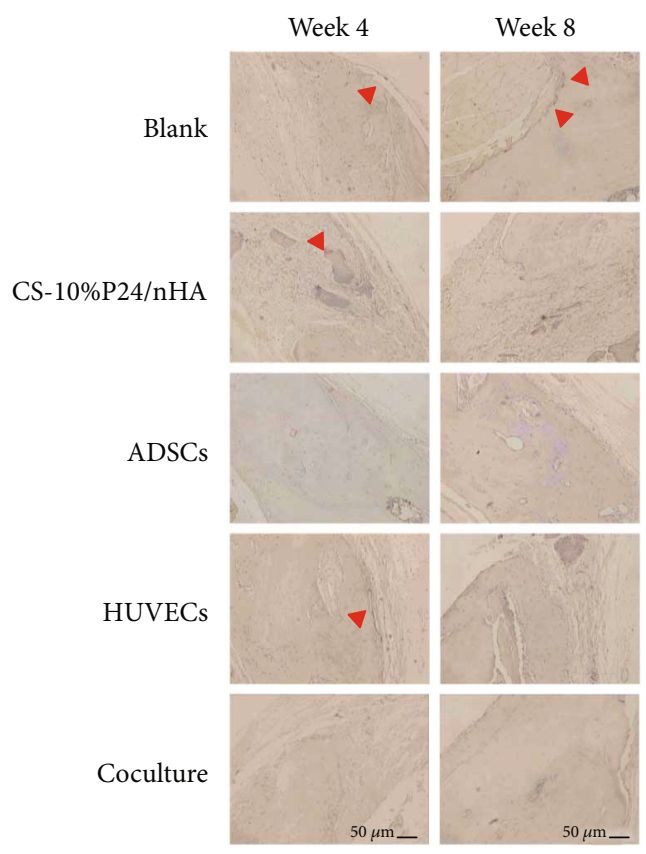

(a)

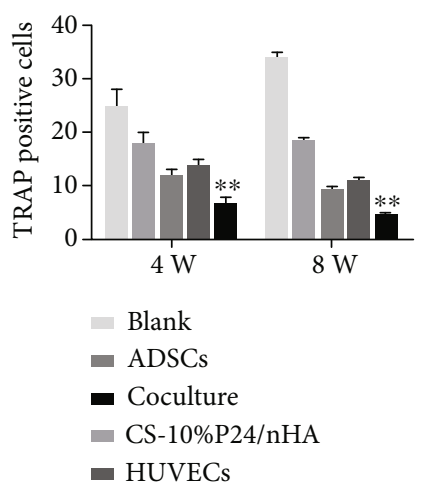

(b)

FIGURE 6: In vivo effects of the scaffold plus ADSC/HUVEC coculture on osteoclast genesis. (a) TRAP staining was performed on three sections per group. (b) Quantification of TRAP-positive cells in each group. Red arrows show TRAP-positive cells. Representative magnified images are at $\times 40$ magnification. Scale bar $=50 \mu \mathrm{m}$.

bone defect repair. Previous studies have observed an interaction between endothelial cells and mesenchymal cells. This interaction promotes proliferation and osteogenic differenti- ation of mesenchymal cells in vitro $[15,24]$. Studies have found that endothelial cells promote MSC differentiation through cell-to-cell contact, which makes cell coculture a 
feasible method [30-32]. These findings encourage research into the effects of endothelial cell and mesenchymal cell coculture to promote bone regeneration.

This study is aimed at determining the effect of HUVE$\mathrm{C} / \mathrm{ADSC}$ coculture on osteogenic differentiation and angiogenesis in CS-P24/nHA scaffolds. We also aimed to explore the optimal proportion of cells needed for coculture and the effect of coculture on osteoporotic bone defect repair. After human ADSC extraction, ADSCs were inoculated with HUVECs on CS-P24/nHA scaffolds to form a coculture system. Compared with separately cultured ADSCs and HUVECs, coculture of HUVECs and ADSCs can significantly promote cell proliferation, which is consistent with the results of previous studies $[13,33]$. To determine the optimal proportion of cells, we cocultured HUVECs and ADSCs at different ratios. The results show that when ADSCs and HUVECs were cocultured at a ratio of $3: 1$, cell proliferation was significantly promoted. These results indicate that the proportion of cocultured cells affects cell behavior. In most studies, a 3:1 ratio of MSCs to HUVECs has achieved good results in promoting cell proliferation and bone regeneration $[10,33-35]$. But in a study by Pennings et al., MSCs and HUVECs were cocultured at a ratio of $4: 1$ to promote bone regeneration [36]. Moreover, Carvalho et al. found that the promoting effects of coculture were best at a ratio of $1: 1$ [37]. However, Shah et al. found that a HUVEC to MSC ratio of $5: 1$ significantly promoted angiogenesis, while a HUVEC to MSC ratio of 1:5 effectively promoted osteogenesis and mineralization [38]. Given the above discrepancies, we cocultured these two cell types at a ratio of $3: 1$ and detected osteogenic differentiation of ADSCs. PCR showed that coculture at a ratio of $3: 1$ had the most obvious effect on osteogenic differentiation and significantly increased the expression of osteogenesis-related genes, including RUNX2, $O C N$, and $A L P$. In our study, 3:1 was the most suitable ratio for coculture of endothelial cells and stem cells, and this ratio significantly promoted cell proliferation and osteogenic differentiation. Despite these observations, there is no unified standard for the best proportion of cocultured cells. Thus, this area is worthy of further research.

To verify the effect of coculture on osteoporotic bone defect repair, ADSCs and HUVECs were inoculated on CSP24/nHA scaffolds at a ratio of 3:1 and implanted to repair calvariae defects in osteoporotic rats. Micro-CT showed that CS-P24/nHA scaffolds containing cocultured cells were more effective in repairing osteoporotic bone defects. Histological results also showed that coculture effectively promoted bone regeneration. Thicker regenerated skulls and better bone remodeling were observed in the coculture group compared with the single-cell groups. We also observed that the defect was only filled with fibrous tissue in the blank and scaffold groups, which indicated that bone regeneration was significantly less evident. This may be due to inhibition of osteoblast activity in osteoporotic animals, resulting in a poor effect of simple scaffold filling on bone defect repair $[29,39]$. Our results proved that the combination of nanoscaffolds and cell coculture can improve the effect of scaffolds on bone defect repair. Coculture of HUVECs and ADSCs in the scaffold can provide seed cells for bone repair and pro- mote vascular regeneration by endothelial cells, especially in the context of osteoporotic bone defect repair. However, the CS-TBA nanocomposite scaffold not only provided an appropriate external environment for HUVECs and ADSCs but also promoted the proliferation and differentiation of cells by releasing P24 and mechanical stress [40, 41]. The combination of nanomaterial and cell coculture improves the therapeutic effect, and this combination method is helpful to explore more improved methods of nanomaterials.

In addition to promoting bone regeneration, inhibiting bone resorption is important for osteoporotic bone defect repair $[4,5]$. Therefore, we use TRAP staining to further explore the effect of coculture scaffolds on the activity of osteoclasts in bone defects. The results show that the number of osteoclasts at the defect site was reduced in the group that received the scaffold plus ADSC/HUVEC coculture. The results show that coculture does not only promote bone regeneration but that it also inhibits bone resorption, which is essential for osteoporotic bone defect repair. Other studies have shown that coculture of endothelial cells and mesenchymal cells can inhibit osteoclast activity [26, 42, 43]. However, some studies suggest that cocultured microvessels promote osteoclast proliferation and activity [44]. In our study, cell coculture inhibited osteoclast activity at the defect site, which may be due to the activity of HUVECs. Alternatively, other cell-to-cell interactions may have played a role, which should be clarified in the future.

\section{Conclusion}

This study demonstrated that coculture of HUVECs and ADSCs in CS-P24/nHA nanocomposite scaffolds can promote cell proliferation and osteogenic differentiation. When ADSCs and HUVECs were cocultured at a ratio of $3: 1$, their effect on proliferation and osteogenic differentiation was the most obvious. Using a rodent model of osteoporotic bone defect, we further confirmed that coculture of HUVECs and ADSCs with the CS-P24/nHA nanocomposite scaffold promoted bone defect repair and inhibited osteoclast activity at the defect site. Our research shows that the CS-P24/nHA nanocomposite scaffold plus ADSC/HUVEC coculture can effectively repair osteoporotic bone defects, which has broad application and development prospects in the field of orthopedics.

\section{Data Availability}

The generated or analyzed data used to support the findings of this study are included within the article.

\section{Conflicts of Interest}

The authors have no conflicts of interest to declare.

\section{Acknowledgments}

This work was supported by the National Natural Science Foundation of China (grant numbers: 81371931, 81974323, and 81301240), the Natural Science Foundation of Guangdong Province, China (grant numbers: 2014A030313352, 
and 2014A030313310), the Shenzhen Strategic Emerging Industries Project (grant number: CXZZ20130517095548798), the Guangzhou Science and Technology Program Project (grant number: 201804010082), and the Special Funds for the Cultivation of Guangdong College Students' Scientific and Technological Innovation (grant number: pdjh2016b0097).

\section{References}

[1] A. Sterodimas, J. de Faria, B. Nicaretta, and I. Pitanguy, "Tissue engineering with adipose-derived stem cells (ADSCs): current and future applications," Journal of Plastic, Reconstructive \& Aesthetic Surgery, vol. 63, pp. 1886-1892, 2010.

[2] F. Salamanna, A. Gambardella, D. Contartese, A. Visani, and M. Fini, "Nano-based biomaterials as drug delivery systems against osteoporosis: a systematic review of preclinical and clinical evidence," Nanomaterials, vol. 11, no. 2, 2021.

[3] R. Kocijan, K. Klaushofer, and B. M. Misof, "Osteoporosis Therapeutics 2020," Handbook of Experimental Pharmacology, vol. 262, pp. 397-422, 2020.

[4] M. Lorentzon, "Treating osteoporosis to prevent fractures: current concepts and future developments," Journal of Internal Medicine, vol. 285, no. 4, pp. 381-394, 2019.

[5] L. Aghebati-Maleki, S. Dolati, R. Zandi et al., "Prospect of mesenchymal stem cells in therapy of osteoporosis: a review," Journal of Cellular Physiology, vol. 234, no. 6, pp. 8570-8578, 2019.

[6] C. Zhang and C. Song, "Combination therapy of PTH and antiresorptive drugs on osteoporosis: a review of treatment alternatives," Frontiers in Pharmacology, vol. 11, article 607017, 2020.

[7] A. Saadat, S. Karbasi, A. Ghader, and M. Khodaei, "Characterization of biodegradable $\mathrm{P} 3 \mathrm{HB} / \mathrm{HA}$ nanocomposite scaffold for bone tissue engineering," Procedia Materials Science, vol. 11, pp. 217-223, 2015.

[8] Z. Zou, W. Liu, L. Cao et al., "Advances in the occurrence and biotherapy of osteoporosis," Biochemical Society Transactions, vol. 48, no. 4, pp. 1623-1636, 2020.

[9] A. Qadir, S. Liang, Z. Wu, Z. Chen, L. Hu, and A. Qian, "Senile osteoporosis: the involvement of differentiation and senescence of bone marrow stromal cells," International Journal of Molecular Sciences, vol. 21, no. 1, 2020.

[10] D. N. Heo, M. Hospodiuk, and I. T. Ozbolat, "Synergistic interplay between human MSCs and HUVECs in 3D spheroids laden in collagen/fibrin hydrogels for bone tissue engineering," Acta Biomaterialia, vol. 95, pp. 348-356, 2019.

[11] L. Krishnan, N. J. Willett, and R. E. Guldberg, "Vascularization strategies for bone regeneration," Annals of Biomedical Engineering, vol. 42, no. 2, pp. 432-444, 2014.

[12] C. Seebach, D. Henrich, K. Wilhelm, J. H. Barker, and I. Marzi, "Endothelial progenitor cells improve directly and indirectly early vascularization of mesenchymal stem cell-driven bone regeneration in a critical bone defect in rats," Cell Transplantation, vol. 21, no. 8, pp. 1667-1677, 2012.

[13] I. Kocherova, A. Bryja, P. Mozdziak, A. Angelova Volponi, M. Dyszkiewicz-Konwińska, H. Piotrowska-Kempisty et al., "Human umbilical vein endothelial cells (HUVECs) coculture with osteogenic cells: from molecular communication to engineering prevascularised bone grafts," Journal of Clinical Medicine, vol. 8, 2019.
[14] J. Zhang, K. G. Neoh, and E. T. Kang, "Electrical stimulation of adipose-derived mesenchymal stem cells and endothelial cells co-cultured in a conductive scaffold for potential orthopaedic applications," Journal of Tissue Engineering and Regenerative Medicine, vol. 12, no. 4, pp. 878-889, 2018.

[15] S. M. Goerke, J. Obermeyer, J. Plaha, G. B. Stark, and G. Finkenzeller, "Endothelial progenitor cells from peripheral blood support bone regeneration by provoking an angiogenic response," Microvascular Research, vol. 98, pp. 40-47, 2015.

[16] W. Chen, X. Liu, Q. Chen et al., “Angiogenic and osteogenic regeneration in rats via calcium phosphate scaffold and endothelial cell co-culture with human bone marrow mesenchymal stem cells (MSCs), human umbilical cord MSCs, human induced pluripotent stem cell-derived MSCs and human embryonic stem cell-derived MSCs," Journal of Tissue Engineering and Regenerative Medicine, vol. 12, no. 1, pp. 191203, 2018.

[17] K. Kafedjiiski, F. Föger, M. Werle, and A. Bernkop-Schnürch, "Synthesis and in vitro evaluation of a novel chitosanglutathione conjugate," Pharmaceutical Research, vol. 22, no. 9, pp. 1480-1488, 2005.

[18] A. Matsuda, H. Kobayashi, S. Itoh, K. Kataoka, and J. Tanaka, "Immobilization of laminin peptide in molecularly aligned chitosan by covalent bonding," Biomaterials, vol. 26, no. 15, pp. 2273-2279, 2005.

[19] Y. Chen, X. Liu, R. Liu et al., "Zero-order controlled release of BMP2-derived peptide P24 from the chitosan scaffold by chemical grafting modification technique for promotion of osteogenesisinvitro and enhancement of bone repairin vivo," Theranostics, vol. 7, no. 5, pp. 1072-1087, 2017.

[20] Y. J. Seol, Y. J. Park, S. C. Lee et al., "Enhanced osteogenic promotion around dental implants with synthetic binding motif mimicking bone morphogenetic protein (BMP)-2," Journal of Biomedical Materials Research. Part A, vol. 77, no. 3, pp. 599-607, 2006.

[21] J. Li, J. Hong, Q. Zheng et al., "Repair of rat cranial bone defects with nHAC/PLLA and BMP-2-related peptide or rhBMP-2," Journal of Orthopaedic Research, vol. 29, no. 11, pp. 1745-1752, 2011.

[22] J. Rehman, D. Traktuev, J. Li et al., "Secretion of angiogenic and antiapoptotic factors by human adipose stromal cells," Circulation, vol. 109, no. 10, pp. 1292-1298, 2004.

[23] L. F. Fröhlich, "MicroRNAs at the interface between osteogenesis and angiogenesis as targets for bone regeneration," Cells, vol. 8, no. 2, p. 121, 2019.

[24] Y. Jia, Y. Zhu, S. Qiu, J. Xu, and Y. Chai, “Exosomes secreted by endothelial progenitor cells accelerate bone regeneration during distraction osteogenesis by stimulating angiogenesis," Stem Cell Research \& Therapy, vol. 10, no. 1, p. 12, 2019.

[25] R. Shi, Y. Huang, C. Ma, C. Wu, and W. Tian, "Current advances for bone regeneration based on tissue engineering strategies," Frontiers in Medicine, vol. 13, no. 2, pp. 160-188, 2019.

[26] Y. Yin, Q. Tang, M. Xie, L. Hu, and L. Chen, "Insights into the mechanism of vascular endothelial cells on bone biology," Bioscience Reports, vol. 41, no. 1, 2021.

[27] L. Shang, Y. Xu, C. Shao, C. Ma, and Y. Feng, "Anti-vascular endothelial growth factor (VEGF) antibody ameliorates cartilage degradation in a rat model of chronic sports arthritic injury," Medical Science Monitor, vol. 24, pp. 4073-4079, 2018. 
[28] X. Zhang, R. Crawford, and Y. Xiao, "Inhibition of vascular endothelial growth factor with shRNA in chondrocytes ameliorates osteoarthritis," Journal of Molecular Medicine (Berlin, Germany), vol. 94, no. 7, pp. 787-798, 2016.

[29] U. Föger-Samwald, P. Dovjak, U. Azizi-Semrad, K. KerschanSchindl, and P. Pietschmann, "Osteoporosis: pathophysiology and therapeutic options," EXCLI Journal, vol. 19, pp. 10171037, 2020.

[30] M. Grellier, L. Bordenave, and J. Amédée, "Cell-to-cell communication between osteogenic and endothelial lineages: implications for tissue engineering," Trends in Biotechnology, vol. 27, pp. 562-571, 2009.

[31] B. Guillotin, R. Bareille, C. Bourget, L. Bordenave, and J. Amédée, "Interaction between human umbilical vein endothelial cells and human osteoprogenitors triggers pleiotropic effect that may support osteoblastic function," Bone, vol. 42, no. 6, pp. 1080-1091, 2008.

[32] D. Steiner, F. Lampert, G. B. Stark, and G. Finkenzeller, "Effects of endothelial cells on proliferation and survival of human mesenchymal stem cells and primary osteoblasts," Journal of Orthopaedic Research, vol. 30, pp. 1682-1689, 2012.

[33] X. Liu, W. Chen, C. Zhang et al., "Co-seeding human endothelial cells with human-induced pluripotent stem cell-derived mesenchymal stem cells on calcium phosphate scaffold enhances osteogenesis and vascularization in rats," Tissue Engineering, vol. 23, no. 11-12, Part A, pp. 546-555, 2017.

[34] W. L. Fu, Z. Xiang, F. G. Huang et al., "Coculture of peripheral blood-derived mesenchymal stem cells and endothelial progenitor cells on strontium-doped calcium polyphosphate scaffolds to generate vascularized engineered bone," Tissue Engineering, vol. 21, no. 5-6, Part A, pp. 948-959, 2015.

[35] Y. Xue, Z. Xing, A. I. Bolstad, T. E. Van Dyke, and K. Mustafa, "Co-culture of human bone marrow stromal cells with endothelial cells alters gene expression profiles," The International Journal of Artificial Organs, vol. 36, no. 9, pp. 650-662, 2013.

[36] I. Pennings, L. A. van Dijk, J. van Huuksloot et al., "Effect of donor variation on osteogenesis and vasculogenesis in hydrogel cocultures," Journal of Tissue Engineering and Regenerative Medicine, vol. 13, no. 3, pp. 433-445, 2019.

[37] M. S. Carvalho, J. C. Silva, R. N. Udangawa et al., "Co-culture cell-derived extracellular matrix loaded electrospun microfibrous scaffolds for bone tissue engineering," Materials Science \& Engineering. C, Materials for Biological Applications, vol. 99, pp. 479-490, 2019.

[38] A. R. Shah, J. C. Wenke, and C. M. Agrawal, "Manipulation of human primary endothelial cell and osteoblast coculture ratios to augment vasculogenesis and mineralization," Annals of Plastic Surgery, vol. 77, no. 1, pp. 122-128, 2016.

[39] F. Xu, W. Li, X. Yang, L. Na, L. Chen, and G. Liu, "The roles of epigenetics regulation in bone metabolism and osteoporosis," Frontiers in Cell and Developmental Biology, vol. 8, article 619301, 2020.

[40] L. Wang, S. Wu, G. Cao, Y. Fan, N. Dunne, and X. Li, "Biomechanical studies on biomaterial degradation and co-cultured cells: mechanisms, potential applications, challenges and prospects," Journal of Materials Chemistry B, vol. 7, pp. 7439-7459, 2019.

[41] L. Wang, C. Wang, S. Wu, Y. Fan, and X. Li, "Influence of the mechanical properties of biomaterials on degradability, cell behaviors and signaling pathways: current progress and challenges," Biomaterials Science, vol. 8, pp. 2714-2733, 2020.
[42] S. K. Ramasamy, A. P. Kusumbe, M. Schiller et al., "Blood flow controls bone vascular function and osteogenesis," Nature Communications, vol. 7, no. 1, article 13601, 2016.

[43] S. G. Romeo, K. M. Alawi, J. Rodrigues, A. Singh, A. P. Kusumbe, and S. K. Ramasamy, "Endothelial proteolytic activity and interaction with non-resorbing osteoclasts mediate bone elongation," Nature Cell Biology, vol. 21, no. 4, pp. 430441, 2019.

[44] H. Pang, X. H. Wu, S. L. Fu et al., "Co-culture with endothelial progenitor cells promotes survival, migration, and differentiation of osteoclast precursors," Biochemical and Biophysical Research Communications, vol. 430, no. 2, pp. 729-734, 2013. 